\title{
Schauder estimate for solutions of Poisson's equation with Neumann boundary condition
}

\author{
Giacomo NARDI*
}

\begin{abstract}
We consider here the Neumann problem for the Laplace operator and prove an existence result in the Hölder spaces and obtain Schauder estimates. According to our knowledge this result is not explicitly proved in the several works devoted to Schauder theory, where similar theorems are proved for the Dirichlet and oblique derivative problems. Our contribution is to make explicit the existence and the estimate for the Neumann problem.
\end{abstract}

Mathematics Subject Classification (2010). Primary 35J25, 35J05

Keywords. Schauder estimate, Poisson's equation, Neumann boundary condition

\section{Introduction}

Let $\Omega$ be a $C^{2, \alpha}$-domain of $\mathbb{R}^{N}$ (we refer to Section 2 for notation and definitions). We consider the following problem:

$$
\left\{\begin{aligned}
\Delta u=f & \text { in } \Omega, \\
\frac{\partial u}{\partial n}=g & \text { on } \partial \Omega,
\end{aligned}\right.
$$

with $f \in C^{0, \alpha}(\bar{\Omega})$ and $g \in C^{1, \alpha}(\bar{\Omega})$. The aim of this paper is to prove an existence result in $C^{2, \alpha}(\bar{\Omega})$ for this problem and an estimate of the form

$$
\|u\|_{C^{2, \alpha}} \leq C\left(\|f\|_{C^{0, \alpha}}+\|g\|_{C^{1, \alpha}}\right) .
$$

In the 1930s, this kind of estimate was used by Schauder [Sch] and Caccioppoli [Ca] to prove an existence result in $C^{2, \alpha}(\bar{\Omega})$ for the Dirichlet problem for an elliptic equation ([GT, Theorem 6.8, p. 100]; [LU, Theorem 1.3, p. 107]). Using the same technique, in the 1950s, Fiorenza [Fi] proved a similar estimate and an existence result in $C^{2, \alpha}(\bar{\Omega})$ for the oblique derivative problem

* We thank A. Adimurthi, N. Fusco, R. Gianni, L. Orsina, and N. Trudinger, for their advice. We thank B. Dacorogna who supervised the researches on this subject. 


$$
l(x) u+m(x) \frac{\partial u}{\partial n}=g \text { on } \partial \Omega \quad(\operatorname{lm}>0 \text { on } \partial \Omega)
$$

for elliptic equations ([GT, Theorem 6.31, p. 128]; [LU, Theorem 3.1, p. 126]).

Unfortunately the hypothesis $l \neq 0$ may not be removed in the proof of the existence result and the Schauder estimate for the oblique derivative problem ([GT, Theorem 6.31, p. 128]). Moreover, reading this proof, we can verify that it is not even possible to get the result for the Neumann problem taking the limit $l \rightarrow 0$.

So, the case of the Neumann problem needs to be considered independently and, according to our knowledge, it is not explicitly present in the classical literature on the subject (see for instance [GT], [LU]) where the Dirichlet and oblique derivative problems are studied in detail.

The main goal of this work is to formalize the existence result and a Schauder estimate for the Neumann problem for Poisson's equation.

Our main contribution is the following result (Theorems 3.1 and 4.1):

Theorem. Let $\alpha \in(0,1)$ and let $\Omega$ be a $C^{2, \alpha}$-domain. Let $f \in C^{0, \alpha}(\bar{\Omega})$ and $g \in C^{1, \alpha}(\bar{\Omega})$ be such that

$$
\int_{\Omega} f=\int_{\partial \Omega} g
$$

Then there exists a solution $u \in C^{2, \alpha}(\bar{\Omega})$ (unique up to an additive constant) to the problem

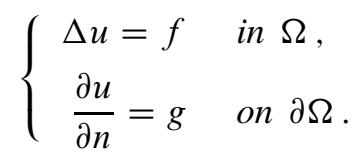

Moreover, every solution to this problem verifies the estimate

$$
\left\|u-\frac{1}{|\Omega|} \int_{\Omega} u\right\|_{C^{2, \alpha}} \leq C\left(\|f\|_{C^{0, \alpha}}+\|g\|_{C^{1, \alpha}}\right),
$$

with $C=C(\Omega, N, \alpha)$.

The starting point of the proof is the alternative theorem for the oblique derivative problem for an uniformly elliptic operator $L$ with coefficients in $C^{0, \alpha}(\bar{\Omega})$ and $c<0$ (Theorem 5.1). This allows us to prove an existence result (uniqueness is given up to a constant) for the Neumann problem for elliptic operators with $c<0$ (Theorem 5.2). Afterwards, using Theorem 5.2 and the Fredholm alternative, we can prove the existence and uniqueness of the solution to our initial problem in the class of functions belonging to $C^{2, \alpha}(\bar{\Omega})$ and having null average (Theorem 3.1). 
Concerning the estimate we are led to estimate the quantity $u-\frac{1}{|\Omega|} \int u$ instead of $u$, because the solution to the Neumann problem, if it exists, is unique up to a constant. We give three proofs of this result (Theorem 4.1).

We finally obtain, for the Neumann problem, results similar to those for the Dirichlet and oblique derivative boundary conditions.

As already mentioned, the theorem is well known to all specialists in elliptic partial differential equations. However, after discussion with several of them, we were not able to find a precise reference for such a result, specially the one concerning the estimate.

This paper is organized as follows. In Section 2 we recall the main definitions used in this work. In Section 3 we prove an existence theorem for the Neumann problem for the Poisson equation (Theorem 3.1). In Section 4 we prove the estimate (Theorem 4.1). In Section 5 we recall some useful results to prove the existence theorem. We adopt the same notation as in [GT]. We refer to [Na] for a more detailed analysis of the problem.

\section{Definitions and notation}

In the sequel we denote by $\Omega$ an open bounded non-empty subset of $\mathbb{R}^{N}(N \geq 2)$ and let $u$ be a function defined on $\Omega$. For every multi-index $\beta=\left(\beta_{1}, \ldots, \beta_{N}\right)\left(\beta_{i} \geq 0\right.$ for $\left.i=1, \ldots, N\right)$ of length $|\beta|=\sum_{i=1}^{N} \beta_{i}$, we set

$$
D^{\beta} u=\frac{\partial^{|\beta|} u}{\partial x_{1}^{\beta_{1}} \ldots \partial x_{N}^{\beta_{N}}} .
$$

We recall the definition of the usual functional spaces $(k \geq 0)$ :

$$
\begin{gathered}
C^{k}(\Omega)=\left\{u: \Omega \rightarrow \mathbb{R} \mid \forall \beta \text { multi-index, }|\beta| \leq k, D^{\beta} u \text { is continuous in } \Omega\right\}, \\
C^{k}(\bar{\Omega})=\left\{u \in C^{k}(\Omega)\left|D^{\beta} u,\right| \beta \mid \leq k, \text { can be extended by continuity to } \partial \Omega\right\} .
\end{gathered}
$$

Moreover $C^{k}(\bar{\Omega})$ is a Banach space equipped with the norm

$$
\|u\|_{C^{k}}=\sum_{i=0}^{k} \sup _{|\beta|=i} \sup _{\bar{\Omega}}\left|D^{\beta} u\right| .
$$

We now recall the definition of Hölder spaces.

Definition 2.1 (Hölder-continuity). Let $\alpha \in] 0,1]$ and let $\Omega \subset \mathbb{R}^{N}$ be an open set. We define the $\alpha$-Hölder coefficient of $u: \Omega \rightarrow \mathbb{R}$ as

$$
[u]_{0, \alpha ; \Omega}=\sup _{\substack{x, y \in \Omega \\ x \neq y}} \frac{|u(x)-u(y)|}{|x-y|^{\alpha}} .
$$

If $[u]_{0, \alpha ; \Omega}<\infty$ then we say that $u$ is Hölder continuous with exponent $\alpha$ in $\Omega$. If there is no ambiguity about the domain $\Omega$ we denote $[u]_{0, \alpha ; \Omega}$ by $[u]_{0, \alpha}$. 
We define the Hölder space $C^{k, \alpha}(\bar{\Omega})$ as the set of functions belonging to $C^{k}(\bar{\Omega})$ whose $k$ th-order partial derivatives are Hölder continuous with exponent $\alpha$ in $\Omega$.

$C^{k, \alpha}(\bar{\Omega})$ is a Banach space equipped with the following norm:

$$
\|u\|_{C^{k, \alpha}}=\|u\|_{C^{k}}+[u]_{k, \alpha},
$$

where

$$
[u]_{k, \alpha}=\sup _{|\beta|=k}\left[D^{\beta} u\right]_{0, \alpha}
$$

We set $C^{k, 0}(\bar{\Omega})=C^{k}(\bar{\Omega})$, and one can easily verify that $C^{k, \alpha}(\bar{\Omega}) \subset C^{h, \alpha}(\bar{\Omega})$ for all integers $h, k$ with $h<k$.

Moreover, for every vector-valued function $u: \Omega \rightarrow \mathbb{R}^{N}$, we say that it belongs to $C^{k, \alpha}(\bar{\Omega})$ if its components belong to $C^{k, \alpha}(\bar{\Omega})$.

In order to give the definition of $C^{k, \alpha}$-domains, we need the following notation:

$$
\begin{gathered}
B=\left\{x=\left(x_{1}, \ldots, x_{N}\right) \in \mathbb{R}^{N}|| x \mid<1\right\}, \\
B_{+}=\left\{x \in B \mid x_{N}>0\right\}, \quad B_{0}=\left\{x \in B \mid x_{N}=0\right\} .
\end{gathered}
$$

Definition 2.2. We call domain every open, bounded, connected, and non-empty subset $\Omega$ of $\mathbb{R}^{N}$. Moreover, $\Omega$ is said to be a $C^{k, \alpha}$-domain $(k \geq 1, \alpha \in[0,1])$ if for every $p \in \partial \Omega$ there exists a neighborhood $U_{p}$ of $p$ in $\mathbb{R}^{N}$ and a diffeomorphism $\varphi_{p}: B \rightarrow U_{p}$ such that

(i) $\varphi_{p} \in C^{k, \alpha}(\bar{B})$ and $\varphi_{p}^{-1} \in C^{k, \alpha}\left(\overline{U_{p}}\right)$;

(ii) $\varphi_{p}\left(B_{+}\right)=U_{p} \cap \Omega$;

(iii) $\varphi_{p}\left(B_{0}\right)=U_{p} \cap \partial \Omega$.

Remark 2.3 (Hölder-continuity on the boundary). Let $\Omega$ be a $C^{k, \alpha}$-domain. We say that $u \in C^{k, \alpha}(\partial \Omega)$ if, for every $p \in \partial \Omega$, we have $u \circ \varphi_{p} \in C^{k, \alpha}\left(B_{0}\right)$, where $\varphi_{p}$ is given by the previous definition.

Of course if $u \in C^{k, \alpha}(\bar{\Omega})$ its restriction to $\partial \Omega$ belongs to $C^{k, \alpha}(\partial \Omega)$. Moreover, for every $u \in C^{k, \alpha}(\partial \Omega)$ there exists a function belonging to $C^{k, \alpha}(\bar{\Omega})$ whose restriction to the boundary coincides with $u$ (see [GT]: Lemma 6.38 p. 137).

Then, in order to study the Neumann problem for Poisson's equation, we can consider boundary values belonging to $C^{k, \alpha}(\bar{\Omega})$ instead of $C^{k, \alpha}(\partial \Omega)$.

We denote by $L^{p}$ and $W^{m, p}$ the usual Lebesgue and Sobolev spaces and refer to $[\mathrm{AF}, \mathrm{Br}]$ for their properties. 


\section{Existence of solutions to the Neumann problem for Poisson's equation in $C^{2, \alpha}(\bar{\Omega})$}

In this section we consider Poisson's equation with Neumann boundary condition and prove the following result:

Theorem 3.1. Let $\Omega$ be a $C^{2, \alpha}$-domain and let $f \in C^{0, \alpha}(\bar{\Omega}), g \in C^{1, \alpha}(\bar{\Omega})$ be such that

$$
\int_{\Omega} f=\int_{\partial \Omega} g
$$

Then the problem

$$
\begin{cases}\Delta u=f & \text { in } \Omega, \\ \frac{\partial u}{\partial n}=g & \text { on } \partial \Omega,\end{cases}
$$

admits a unique solution in the class

$$
\mathcal{C}=\left\{u \in C^{2, \alpha}(\bar{\Omega}): \frac{1}{|\Omega|} \int_{\Omega} u=0\right\} .
$$

We start by recalling the following estimate which is very useful in the sequel:

Theorem 3.2 ([GT, Theorem 6.30, p. 127]; [LU, Theorem 3.1, p. 126]). Let $\Omega$ be a $C^{2, \alpha}$-domain and let $u \in C^{2, \alpha}(\bar{\Omega})$ be a solution of (3.2). Then

$$
\|u\|_{C^{2, \alpha}} \leq C\left(\|f\|_{C^{0, \alpha}}+\|g\|_{C^{1, \alpha}}+\|u\|_{C^{0}}\right),
$$

with $C=C(\Omega, \alpha, N)$.

We prove two preliminary lemmas.

Lemma 3.3. Let $\Omega \subset \mathbb{R}^{N}$ be a $C^{2}$-domain and let $u \in C^{2}(\bar{\Omega})$. We suppose that there exists $p \in \partial \Omega$ such that:

$$
u(p)=\max _{\bar{\Omega}} u\left(\min _{\bar{\Omega}} u\right) \text { and } D u(p)=0 .
$$

Then $\Delta u(p) \leq 0(\geq 0)$.

Proof. Up to a translation, we may assume that $p$ is the origin of $\mathbb{R}^{N}$. Moreover, as $\Omega \in C^{2}$, up to a rotation, we may also assume that, for some $R$, we have $-r e_{i} \in \Omega$ for every $r \in[0, R]$ and $i=1, \ldots, N$ where $\left(e_{1}, \ldots, e_{N}\right)$ is an orthonormal basis of $\mathbb{R}^{N}$. Then, partial derivatives verify

$$
D_{i i} u(p)=\lim _{h \rightarrow 0^{-}} \frac{D_{i} u\left(p+h e_{i}\right)-D_{i} u(p)}{h}=\lim _{h \rightarrow 0^{-}} \frac{D_{i} u\left(p+h e_{i}\right)}{h} \leq 0,
$$

because $D u(p)=0$ and, for $h<0$ small enough we have $D_{i} u\left(p+h e_{i}\right) \geq 0$. If $p$ is a minimum point the proof is similar. 
Lemma 3.4. Let $\Omega \subset \mathbb{R}^{N}$ be a $C^{2}$-domain and $f \in C^{0, \alpha}(\bar{\Omega})$. Let $u \in C^{2, \alpha}(\bar{\Omega})$ be a solution to the problem

$$
\left\{\begin{array}{rlrl}
\Delta u-u & =f & \text { in } \Omega \\
\frac{\partial u}{\partial n} & =0 & & \text { on } \partial \Omega
\end{array}\right.
$$

then

$$
\|u\|_{C^{0}} \leq\|f\|_{C^{0}}
$$

Proof. Let $p$ be such that $|u(p)|=\max _{\bar{\Omega}}|u|$. We consider separately the following cases: $p \in \Omega$ and $p \in \partial \Omega$.

$p \in \Omega$. Let $p$ be a maximum point for $u$ then $u(p) \geq 0$ and $\Delta u(p) \leq 0$. As $\Delta u-u=f$ we get $f(p)=\Delta u(p)-u(p) \leq \Delta u(p) \leq 0$. Thus

$$
\|u\|_{C^{0}}=u(p)=\Delta u(p)-f(p) \leq-f(p) \leq\|f\|_{C^{0}} .
$$

If $p$ is a minimum point for $u(u(p) \leq 0)$ the proof is similar.

$p \in \partial \Omega$. If $p$ is a maximum point for $u$ then $p$ is a maximum point for $u_{\mid \partial \Omega}$. So, for every vector $\tau$ tangent to $\partial \Omega$ at $p$ we have:

$$
\frac{\partial u}{\partial \tau}(p)=0
$$

and, using the hypothesis

$$
\frac{\partial u}{\partial n}(p)=0
$$

we get $D u(p)=0$. Thanks to the previous lemma we have $\Delta u(p) \leq 0$ and, as $\Delta u-u=f$ on $\partial \Omega$, the lemma ensues. We can use the same arguments if $p$ is a minimum point for $u$.

Now, we can prove the existence theorem for the Neumann problem for the Laplace operator:

Proof of Theorem 3.1. By Theorem 5.2 there exists a unique solution in $C^{2, \alpha}(\bar{\Omega})$, denoted by $\mathfrak{I}[f, g]$, to the problem:

$$
\left\{\begin{aligned}
\Delta u-u & =f & & \text { in } \Omega, \\
\frac{\partial u}{\partial n} & =g & & \text { on } \partial \Omega,
\end{aligned}\right.
$$

for all $f, g$ verifying the compatibility condition (3.1). Moreover

$$
\int_{\Omega} u=\int_{\Omega} \Delta u-\int_{\Omega} f=\int_{\partial \Omega} \frac{\partial u}{\partial n}-\int_{\partial \Omega} g=0,
$$

so $\mathfrak{I}[f, g] \in \mathcal{C}$. Defining 


$$
\mathcal{A}=\left\{(f, g) \in C^{0, \alpha}(\bar{\Omega}) \times C^{1, \alpha}(\bar{\Omega}): \int_{\Omega} f=\int_{\partial \Omega} g\right\}
$$

we have that $\mathfrak{I}: \mathcal{A} \rightarrow \mathcal{C}$ is a well defined bijective operator.

Now, we consider the following equation:

$$
u-\mathfrak{I}[-u, 0]=\mathfrak{I}[f, g] .
$$

Then $u \in \mathcal{C}$ is a solution to the problem (3.2) if and only if it is a solution of (3.5), because

$$
(f, g)=\mathfrak{I}^{-1}(u-\mathfrak{I}[-u, 0])=\mathfrak{I}^{-1} u+(u, 0)=\left(\Delta u, \frac{\partial u}{\partial n}\right) .
$$

Then, we need to show that for every $(f, g) \in \mathcal{A}$ there exists a unique solution $u \in \mathcal{C}$ of (3.5). As $\mathfrak{I}$ is bijective, we are led to prove that, for every $v \in \mathcal{C}$, the equation

$$
u-\mathfrak{I}[-u, 0]=v
$$

admits a unique solution on $\ell$. For this we use the Fredholm alternative theorem.

We consider the space

$$
\mathcal{F}=\left\{f \in C^{0, \alpha}(\bar{\Omega}): \frac{1}{|\Omega|} \int_{\Omega} f=0\right\},
$$

equipped with the norm of $C^{0, \alpha}(\bar{\Omega})$. Let $T$ be the operator:

$$
\begin{gathered}
T: \mathcal{F} \rightarrow \mathcal{F}, \\
T f=\mathfrak{I}[-f, 0] .
\end{gathered}
$$

Using the properties of $\mathfrak{I}$, we get:

$$
T(\mathcal{F}) \subset \mathcal{C} .
$$

We first show that $T$ is a compact operator. Let $\left\{f_{k}\right\} \subset \mathcal{F}$ then, because of Theorem 5.2 and (3.4), there exists $\left\{u_{k}\right\} \subset \mathcal{C}$ such that $T f_{k}=u_{k}$ and, because of (3.3), we have

$$
\left\|u_{k}\right\|_{C^{2, \alpha}} \leq C(\Omega, \alpha, N)\left(\left\|f_{k}\right\|_{C^{0, \alpha}}+\left\|u_{k}\right\|_{C^{0}}\right) .
$$

So, using the previous lemma, we get:

$$
\left\|u_{k}\right\|_{C^{2, \alpha}} \leq 2 C(\Omega, \alpha, N)\left\|f_{k}\right\|_{C^{0, \alpha}} .
$$

If $\left\{f_{k}\right\}$ is a bounded sequence of $\mathcal{F}$ then $\left\{u_{k}\right\}$ is bounded in $C^{2, \alpha}(\bar{\Omega})$ and in $W^{2, \infty}(\Omega)$. Thus there exists a subsequence $\left\{u_{k_{h}}\right\}$ and a function $u \in W^{2, \infty}(\Omega)$ such that 


$$
u_{k_{h}} \stackrel{*}{\rightarrow} u \text { in } W^{2, \infty},
$$

so, for every $p>1$

$$
u_{k_{h}} \stackrel{*}{\rightarrow} u \text { in } W^{2, p}
$$

Now, choosing $\frac{N}{2-\alpha}<p \leq N$, by the Rellich-Kondrachov theorem, we obtain

$$
u_{k_{h}} \rightarrow u \text { in } C^{0, \alpha}(\bar{\Omega})
$$

which proves that $T$ is compact.

Now, equation (3.6) can be rewritten as

$$
u-T u=v .
$$

The equation

$$
u-T u=0
$$

is equivalent to the problem

$$
\left\{\begin{array}{l}
\Delta u=0 \quad \text { in } \Omega, \\
\frac{\partial u}{\partial n}=0 \quad \text { on } \partial \Omega,
\end{array}\right.
$$

which, in $\ell$, admits only the trivial solution $u=0$.

Then, as $T$ is compact, applying the Fredholm alternative to (3.8) we have that for every $v \in \mathcal{C}$ there exists a unique solution $u \in \mathcal{C}$ of (3.8) and the theorem ensues.

Remark 3.5. If $u \in \mathcal{C}$ is a solution to the problem (3.2) then, for every $k \in \mathbb{R}$, the function $u+k$ is also a solution to (3.2) in $C^{2, \alpha}(\bar{\Omega})$ (but not in $\mathcal{C}$ ).

On the other hand, if $u$ is a solution to (3.2) in $C^{2, \alpha}(\bar{\Omega})$, we can obtain a solution in $\varphi$ setting

$$
v=u-\frac{1}{|\Omega|} \int_{\Omega} u
$$

Then, using Theorem 3.1 we have an existence and uniqueness (up to an additive constant) result for the problem (3.2) in $C^{2, \alpha}(\bar{\Omega})$.

\section{Schauder estimate}

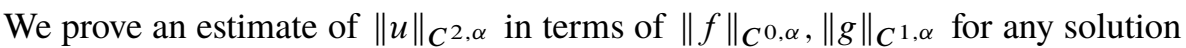
to problem (3.2). In particular, as the uniqueness of the solution is proved up to an additive constant, we prove the estimate for a solution with null average.

We state the following theorem and give three proofs: 
Theorem 4.1. Let $\Omega$ be a $C^{2, \alpha}$-domain and $f \in C^{0, \alpha}(\bar{\Omega}), g \in C^{1, \alpha}(\bar{\Omega})$ be such that

$$
\int_{\Omega} f=\int_{\partial \Omega} g .
$$

Let $u \in C^{2, \alpha}(\bar{\Omega})$ be a solution to (3.2). Then

$$
\left\|u-\frac{1}{|\Omega|} \int_{\Omega} u\right\|_{C^{2, \alpha}} \leq C\left(\|f\|_{C^{0, \alpha}}+\|g\|_{C^{1, \alpha}}\right),
$$

with $C=C(\Omega, \alpha, N)$.

The first proof of Theorem 4.1 was suggested to us by A. Adimurthy who attributed it to Jacques-Louis Lions. However we did not find any reference for such a proof, which is detailed in the sequel.

First proof of Theorem 4.1. Let $u \in C^{2, \alpha}(\bar{\Omega})$ be a solution to (3.2) such that $\frac{1}{\Omega \mid} \int_{\Omega} u=0$. By (3.3), we have the following estimate for $u$ :

$$
\|u\|_{C^{2, \alpha}} \leq C_{1}(\Omega, \alpha, N)\left(\|u\|_{C^{0}}+\|f\|_{C^{0, \alpha}}+\|g\|_{C^{1, \alpha}}\right),
$$

and we wish to prove that

$$
\|u\|_{C^{2, \alpha}} \leq C_{2}(\Omega, \alpha, N)\left(\|f\|_{C^{0, \alpha}}+\|g\|_{C^{1, \alpha}}\right) .
$$

Let us suppose that (4.2) is false. Then for every $k \in \mathbb{N}$ there exist $\left\{u_{k}\right\} \in C^{2, \alpha}(\bar{\Omega})$ and $\left\{f_{k}\right\} \in C^{0, \alpha}(\bar{\Omega}),\left\{g_{k}\right\} \in C^{1, \alpha}(\bar{\Omega})$ such that

$$
\begin{gathered}
\left\{\begin{array}{c}
\Delta u_{k}=f_{k} \quad \text { in } \Omega, \\
\frac{\partial u_{k}}{\partial n}=g_{k} \quad \text { on } \partial \Omega, \\
\frac{1}{|\Omega|} \int_{\Omega} u_{k}=0, \\
\left\|u_{k}\right\|_{C^{2, \alpha}}=1,
\end{array}\right. \\
\left\|u_{k}\right\|_{C^{2, \alpha}}>k\left(\left\|f_{k}\right\|_{C^{0, \alpha}}+\left\|g_{k}\right\|_{C^{1, \alpha}}\right) .
\end{gathered}
$$

Thus, we have $f_{k} \rightarrow 0$ in $C^{0, \alpha}(\bar{\Omega})$ and $g_{k} \rightarrow 0$ in $C^{1, \alpha}(\bar{\Omega})$.

Using (4.5) we have, that for every multi-index $\beta,|\beta|=0,1,2,\left\{D^{\beta} u_{k}\right\}$ is uniformly bounded in $C^{0}(\bar{\Omega})$ and equicontinuous because

$$
\left|D^{\beta} u_{k}(x)-D^{\beta} u_{k}(y)\right| \leq|x-y|^{\alpha} \quad \forall x, y \in \Omega, \forall|\beta|=2,
$$

which implies that

$$
\left|D^{\beta} u_{k}(x)-D^{\beta} u_{k}(y)\right| \leq C(\Omega)|x-y|^{\alpha} \quad \forall x, y \in \Omega, \forall|\beta|=0,1 .
$$


Iterating the Ascoli-Arzelà theorem we get a subsequence $\left\{u_{k_{h}}\right\}$ such that

$$
\begin{aligned}
& u_{k_{h}} \rightarrow u_{0} \text { in } C^{0}(\bar{\Omega}), \\
& D^{\beta} u_{k_{h}} \rightarrow u_{\beta} \text { in } C^{0}(\bar{\Omega}) \quad \forall \beta,|\beta|=1,2,
\end{aligned}
$$

which implies that

$$
u_{k_{h}} \rightarrow u_{0} \text { in } C^{2}(\bar{\Omega})
$$

Then

$$
\begin{aligned}
& \Delta u_{0}=\lim _{h} \Delta u_{k_{h}}=\lim _{h} f_{k_{h}}=0, \\
& \frac{\partial u_{0}}{\partial n}=\lim _{h} \frac{\partial u_{k_{h}}}{\partial n}=\lim _{h} g_{k_{h}}=0,
\end{aligned}
$$

and

$$
\left\{\begin{array}{rlr}
\Delta u_{0} & =0 & \text { in } \Omega, \\
\frac{\partial u_{0}}{\partial n} & =0 & \text { on } \partial \Omega, \\
\frac{1}{|\Omega|} \int_{\Omega} u_{0} & =0, &
\end{array}\right.
$$

which implies that $u_{0}=0$. Comparing with (4.1), we get a contradiction because

$$
1=\left\|u_{k_{h}}\right\|_{C^{2, \alpha}} \leq C_{1}(\Omega, \alpha, N)\left(\left\|u_{k_{h}}\right\|_{C^{0}}+\left\|f_{k_{h}}\right\|_{C^{0, \alpha}}+\left\|g_{k_{h}}\right\|_{C^{1, \alpha}}\right) \rightarrow 0 .
$$

For the second proof of Theorem 4.1 we need two more lemmas. The first one states an estimate in $L^{2}(\Omega)$ for $u-\frac{1}{|\Omega|} \int_{\Omega} u$ :

Lemma 4.2. Let $\Omega$ be a $C^{2, \alpha}$-domain and let $f \in C^{0, \alpha}(\bar{\Omega}), g \in C^{1, \alpha}(\bar{\Omega})$ be such that

$$
\int_{\Omega} f=\int_{\partial \Omega} g
$$

Let $u \in C^{2, \alpha}(\bar{\Omega})$ be a solution to (3.2). Then

$$
\left\|u-\frac{1}{|\Omega|} \int_{\Omega} u\right\|_{L^{2}} \leq C\left(\|f\|_{C^{0, \alpha}}+\|g\|_{C^{1, \alpha}}\right),
$$

with $C=C(\Omega, N)$.

Proof. We may assume that $\frac{1}{|\Omega|} \int_{\Omega} u=0$. Integrating by parts we have

$$
\int_{\Omega}|D u|^{2}=\int_{\partial \Omega} u g-\int_{\Omega} u f
$$

and using Young's inequality with $\varepsilon>0$ we get 
$\int_{\Omega}|D u|^{2} \leq \int_{\partial \Omega}|u g|+\int_{\Omega}|u f| \leq \varepsilon \int_{\partial \Omega}|u|^{2}+\frac{1}{4 \varepsilon} \int_{\partial \Omega}|g|^{2}+\varepsilon \int_{\Omega}|u|^{2}+\frac{1}{4 \varepsilon} \int_{\Omega}|f|^{2}$.

Now, we have

$$
\int_{\partial \Omega}|u|^{2} \leq C_{1}(\Omega)\|u\|_{W^{1,2}}^{2} \quad \forall u \in W^{1,2}(\Omega) \cap C(\bar{\Omega}) ;
$$

so

$$
\int_{\Omega}|D u|^{2} \leq \varepsilon C_{2}(\Omega)\|u\|_{W^{1,2}}^{2}+C_{3}(\Omega, \varepsilon)\left[\|g\|_{W^{1,2}}^{2}+\|f\|_{L^{2}}^{2}\right]
$$

and, as $u \in \mathcal{C}$, using the Poincaré inequality, we get

$$
\int_{\Omega}|D u|^{2} \leq \varepsilon C_{4}(\Omega, N)\|D u\|_{L^{2}}^{2}+C_{5}(\Omega, \varepsilon)\left[\|g\|_{C^{1, \alpha}}^{2}+\|f\|_{C^{0, \alpha}}^{2}\right] .
$$

Choosing $\varepsilon<1 / C_{4}(\Omega, N)$ and using the Poincaré inequality we have

$$
\|u\|_{L^{2}} \leq C_{6}(\Omega, N)\|D u\|_{L^{2}} \leq C\left(\|g\|_{C^{1, \alpha}}+\|f\|_{C^{0, \alpha}}\right),
$$

with $C=C(\Omega, N)$.

The second lemma proves a local estimate for a solution of Poisson's equation:

Lemma 4.3 ([Se, Theorem 1, p. 255, and Theorem 2, p. 259]). Let $\Omega$ be a domain of $\mathbb{R}^{N}$. Let $f \in L^{p}(\Omega)$ with $p>N / 2$ and $u \in C^{2}(\Omega)$ be a solution of $\Delta u=f$ in $\Omega$. Then, for every ball $B(y, 2 R) \subset \Omega$, we have

$$
\sup _{B(y, R)}|u| \leq C\left(R^{-\frac{N}{2}}\|u\|_{L^{2}(B(y, 2 R))}+R^{2-\frac{N}{p}}\|f\|_{L^{p}(\Omega)}\right),
$$

with $C=C(N, p)$.

Following an idea of N. Fusco, we can now give the second proof of Theorem 4.1.

Second proof of Theorem 4.1. We may assume that $\frac{1}{|\Omega|} \int_{\Omega} u=0$. We give an estimate for $\sup _{\partial \Omega}|u|$. As $\Omega$ is a $C^{2, \alpha}$-domain, for every small enough $\varepsilon$ we have

$$
(x-\varepsilon n(x)) \in \Omega \quad \forall x \in \partial \Omega
$$

where $n(x)$ denotes the unit outer-pointing normal to $\partial \Omega$ at $x$. Then, by the Lagrange theorem, for every $x \in \partial \Omega$ there exists $\tau=\tau(x, \varepsilon) \in(0,1)$ such that

$$
u(x-\varepsilon n(x))=u(x)+(D u(x-\tau \varepsilon n(x)),-\varepsilon n(x)) .
$$

Defining $\Omega_{\varepsilon}=\{x \in \Omega \mid \operatorname{dist}(x, \partial \Omega) \geq \varepsilon\}$ and taking the supremum for $x \in \partial \Omega$ in the previous relationship we get: 


$$
\sup _{\partial \Omega}|u| \leq \sup _{\Omega_{\varepsilon}}|u|+\varepsilon \sup _{\Omega}|D u|
$$

Let $R<\varepsilon / 2$. We consider a finite cover of $\Omega_{\varepsilon}$, denoted by $\left\{B\left(y_{i}, R\right)\right\}_{i=1}^{M}$ with $M=M(\varepsilon, \Omega)\left(B\left(y_{i}, R\right)\right.$ denotes an open ball of radius $R$ centered at $\left.y_{i}\right)$. As $f \in C^{0}(\bar{\Omega})$ then $f \in L^{N+1}(\Omega)$ and, by Lemma 4.3, we have

$$
\sup _{\Omega_{\varepsilon}}|u| \leq \sum_{i=1}^{M} \sup _{B\left(y_{i}, R\right)}|u| \leq M C_{1}(N)\left(R^{-\frac{N}{2}}\|u\|_{L^{2}(B(y, 2 R))}+R^{2-\frac{N}{N+1}}\|f\|_{L^{N+1}(\Omega)}\right)
$$

which implies that

$$
\sup _{\Omega_{\varepsilon}}|u| \leq C_{2}(\varepsilon, \Omega, N)\left(\|u\|_{L^{2}}+\|f\|_{L^{N+1}}\right) .
$$

By Theorem 3.7 in [GT, p. 36] and (4.8), we have

$$
\sup _{\Omega}|u| \leq \sup _{\Omega_{\varepsilon}}|u|+\varepsilon \sup _{\Omega}|D u|+C_{3}(\Omega) \sup _{\Omega}|f|
$$

and, by (4.9), we get

$$
\sup _{\Omega}|u| \leq C_{2}(\varepsilon, \Omega, N)\left(\|u\|_{L^{2}}+\|f\|_{L^{N+1}}\right)+\varepsilon \sup _{\Omega}|D u|+C_{3}(\Omega) \sup _{\Omega}|f| .
$$

Using Lemma 4.2, we get

$$
\sup _{\Omega}|u| \leq C_{4}(\varepsilon, \Omega, N)\left(\|f\|_{C^{0, \alpha}}+\|g\|_{C^{1, \alpha}}\right)+\varepsilon\|u\|_{C^{2, \alpha}}
$$

and, by (3.3), we have

$$
\|u\|_{C^{2, \alpha}} \leq C_{5}(\Omega, \alpha, N, \varepsilon)\left(\|f\|_{C^{0, \alpha}}+\|g\|_{C^{1, \alpha}}\right)+\varepsilon C_{6}(\Omega, \alpha, N)\|u\|_{C^{2, \alpha}} .
$$

Choosing $\varepsilon<1 / C_{6}(N, \Omega, \alpha)$ the theorem ensues.

We now give the third proof of Theorem 4.1. We thank R. Gianni who gave us some ideas for this proof.

Third proof of Theorem 4.1. We assume that $\int_{\Omega} u=0$ and, because of (3.3), we just need to prove an estimate for $\|u\|_{C^{0}}$ in terms of $\|f\|_{C^{0, \alpha}}$ and $\|g\|_{C^{1, \alpha}}$.

Let $M=\max _{\bar{\Omega}}|u|$ and $p \in \bar{\Omega}$ be such that $|u(p)|=M$. Moreover, we suppose $M>\left(\|f\|_{C^{0, \alpha}}+\|g\|_{C^{1, \alpha}}\right)$, otherwise there is nothing to prove. By (3.3), we have

$$
\|D u\|_{C^{0}} \leq C_{1}\left(\|f\|_{C^{0, \alpha}}+\|g\|_{C^{1, \alpha}}+\|u\|_{C^{0}}\right)=K,
$$

with $C_{1}=C_{1}(\Omega, \alpha, N)$. We distinguish two cases: $p \in \Omega$ and $p \in \partial \Omega$.

We suppose that $p \in \Omega$ and we denote by $B(p, r)$ the open ball of radius $r$ centered at $p$. We consider in particular $r<r_{0}=\operatorname{dist}(p, \partial \Omega)$ so that such a ball is contained in $\Omega$. 
Now, we can prove that for every $r<r_{0}$, we have

$$
|u|>M-r K, \quad \text { in } B(p, r) .
$$

In fact, for every $x \in B(p, r)$ we have

$$
u(x)-u(p)=\int_{0}^{1} \frac{d}{d t} u(p+t(x-p)) d t=\int_{0}^{1}\langle D u(p+t(x-p)), x-p\rangle d t
$$

so

$$
|u(x)| \geq|u(p)|-K r=M-K r .
$$

Choosing $r<\min \left\{1 / 4 C_{1}, r_{0}\right\}$, for every $x \in B(p, r)$, we have:

$$
\begin{aligned}
|u(x)| \geq M-r K & =\left(1-r C_{1}\right) M-r C_{1}\left(\|f\|_{C^{0, \alpha}}+\|g\|_{C^{1, \alpha}}\right) \\
& >\frac{3}{4} M-r C_{1}\left(\|f\|_{C^{0, \alpha}}+\|g\|_{C^{1, \alpha}}\right) .
\end{aligned}
$$

Then, as $M>\left(\|f\|_{C^{0, \alpha}}+\|g\|_{C^{1, \alpha}}\right)$ and $r<1 / 4 C_{1}$, we get

$$
|u(x)|>\frac{3}{4} M-C_{1} r M>\frac{M}{2} \quad \forall x \in B(p, r)
$$

and, denoting by $\omega_{N}$ the measure of the unit ball of $\mathbb{R}^{N}$, we get

$$
\frac{M}{2} \frac{\omega_{N}^{1 / 2}}{2^{N / 2}} r^{N / 2} \leq\left(\int_{B(p, r)}|u|^{2}\right)^{1 / 2} \leq\|u\|_{L^{2}} .
$$

Then, by Lemma 4.2 , we can state that

$$
\|u\|_{C^{0}} \leq \frac{2^{\frac{N+2}{2}} r^{-\frac{N}{2}}}{\omega_{N}^{1 / 2}} C_{2}(\Omega, N)\left(\|f\|_{C^{0, \alpha}}+\|g\|_{C^{1, \alpha}}\right),
$$

which implies that

$$
\|u\|_{C^{2, \alpha}} \leq C\left(\|f\|_{C^{0, \alpha}}+\|g\|_{C^{1, \alpha}}\right),
$$

with $C=C(\Omega, \alpha, N)$.

We suppose now that $p \in \partial \Omega$. As $\Omega$ is a $C^{2}$-domain, it satisfies the interior sphere condition (see [GT, p. 33]). Then, there exists $r_{0}>0$ such that the open ball $B(p-r n(p), r)$ with $r<r_{0}$ is contained in $\Omega(n(p)$ denotes the unit outer-pointing normal to the boundary at $p$ ).

Thus, as $p$ is not the center of the ball, similarly to the previous case we obtain

$$
|u|>M-2 r K \quad \text { in } B(p-r n(p), r) \quad r<r_{0} .
$$

Then, by choosing $r<\min \left\{1 / 8 C_{1}, r_{0}\right\}$, we get the result by the same argument used above applied to the ball $B(p-r n(p), r)$. 


\section{Appendix: A preliminary result}

In this section we recall some results used in Section 3. We consider a $C^{2, \alpha}$-domain $\Omega$ and the operator

$$
\begin{gathered}
L: C^{2, \alpha}(\bar{\Omega}) \rightarrow \mathbb{R}, \\
L u=\sum_{i, j=1}^{N} a_{i j}(x) \frac{\partial^{2} u}{\partial x_{i} \partial x_{j}}+\sum_{i=1}^{N} b_{i}(x) \frac{\partial u}{\partial x_{i}}+c(x) u,
\end{gathered}
$$

where $a_{i j}, b_{i}, c \in C^{0, \alpha}(\bar{\Omega})$ for every $i, j=1, \ldots, N$ and $c(x) \leq 0$ for every $x \in \bar{\Omega}$.

We say that $L$ is elliptic if the matrix $A(x)=\left[a_{i, j}(x)\right]$ is positive definite for every $x \in \bar{\Omega}$. Moreover, denoting by $\lambda(x)$ the smallest eigenvalue of $A(x)$, we say that $L$ is uniformly elliptic if there exists $\lambda_{0}>0$ such that $\lambda(x) \geq \lambda_{0}$ for every $x \in \bar{\Omega}$.

We refer to [GT, p. 130] for the following alternative result for the oblique derivative problem:

Theorem 5.1. Let $\Omega$ be a $C^{2, \alpha}$-domain. Let $L$ be an elliptic operator, which is uniformly elliptic in $\Omega$ and with $C^{0, \alpha}(\bar{\Omega})$-coefficients. Let $l, m \in C^{1, \alpha}(\bar{\Omega})$ be such that $m \neq 0$ for every $x \in \partial \Omega$. Then exactly one of the following holds:

(1) The homogeneous problem

$$
\left\{\begin{aligned}
L u & =0 & & \text { in } \Omega, \\
l(x) u+m(x) \frac{\partial u}{\partial n} & =0 & & \text { on } \partial \Omega,
\end{aligned}\right.
$$

admits nontrivial solutions;

(2) the homogeneous problem has only the trivial solution, in which case for every $f \in C^{0, \alpha}(\bar{\Omega}), g \in C^{1, \alpha}(\bar{\Omega})$ there exists a solution $u \in C^{2, \alpha}(\bar{\Omega})$ to the inhomogeneous problem

$$
\left\{\begin{aligned}
L u & =f & & \text { in } \Omega, \\
l(x) u+m(x) \frac{\partial u}{\partial n} & =g & & \text { on } \partial \Omega .
\end{aligned}\right.
$$

We can now prove the following theorem:

Theorem 5.2. Let $\Omega$ be $C^{2, \alpha}$-domain. Then for every $f \in C^{0, \alpha}(\bar{\Omega})$ and $g \in C^{1, \alpha}(\bar{\Omega})$ there exists a unique solution $u \in C^{2, \alpha}(\bar{\Omega})$ to the problem

$$
\left\{\begin{aligned}
\Delta u-u & =f & & \text { in } \Omega, \\
\frac{\partial u}{\partial n} & =g & & \text { on } \partial \Omega .
\end{aligned}\right.
$$


Proof. The problem

$$
\left\{\begin{aligned}
\Delta u-u & =0 & & \text { in } \Omega, \\
\frac{\partial u}{\partial n} & =0 & & \text { on } \partial \Omega,
\end{aligned}\right.
$$

admits only the trivial solution. Using Point 2 of Theorem 5.1 the result ensues.

\section{References}

[AF] R. A. Adams and J. J.F. Fournier, Sobolev Spaces. Academic Press, Paris-New York-London, 2003 (Second Edition). Zbl 1098.46001 MR 2424078

[Br] H. Brezis, Analyse Fonctionelle. Masson, Paris-New York-Milan, 1987 ( $2^{e}$ tirage).

[Ca] R. Caccioppoli, Sulle equazioni ellittiche a derivate parziali con $n$ variabili indipendenti. Atti della Accademia Nazionale dei Lincei. Rendiconti Lincei: Matematica e applicazioni. Serie VI 19 (1934), 83-89. Zbl 0009.06802

[Fi] R. Fiorenza, Sui problemi di derivata obliqua per le equazioni ellittiche. Ricerche di Matematica 8 (1959), 83-110. Zbl 0090.31404 MR 0114995

[GT] D. Gilbarg and N. S. Trudinger, Elliptic partial differential equations of second order. Springer-Verlag, Berlin-Heidelberg, 2001 (Reprint of the 1998 edition). Zbl 1042.35002 MR 1814364

[LU] O. A. Ladyženskaja and N. N. Ural'ceva, Equations aux Dérivées Partielles de Type Elliptique. Dunod, Paris, 1968. MR 0239273

[Na] G. NARDI, Risolubilità nel senso classico e nel senso delle distribuzioni per alcuni problemi ellittici. Master thesis, 2007.

[Sch] J.P. Schauder, Über lineare elliptische Differentialgleichungen zweiter Ordnung. Mathematische Zeitschrift B 38 (1934), 257-282. Zbl 0008.25502 MR 1545448

[Se] J. SERrin, Local behavior of solutions of quasi-linear equations. Acta Mathematica 111 (1964), 247-302. Zbl 0128.09101 MR 0170096

(Reçu le 13 février 2014)

Giacomo NARdi, Centre de Recherches en Mathématiques de la Decision (CEREMADE), CNRS : UMR7534, Université Paris IX - Paris Dauphine e-mail:nardi@ceremade.dauphine.fr 